\title{
A chrestomathy on fishes
}

\author{
Darwin's Fishes: An Encyclopedia \\ of Ichthyology, Ecology and \\ Evolution \\ by Daniel Pauly \\ Cambridge University Press: 2004. 366 pp. \\ $\mathfrak{E 5 5 , \$ 8 0}$
}

\section{John C. Avise}

Charles Darwin is renowned for his scientific breadth and prolific writing, as well as his depth of insight. On top of revolutionizing biological thinking (in The Origin of Species and The Descent of Man) and marine geology (in The Structure and Distribution of Coral Reefs), he wrote detailed treatises on creatures ranging from orchids and insectivorous plants to domesticated biotas, barnacles and earthworms. In addition, his name will forever be associated with some other taxonomic groups, notably Darwin's finches. During his career, Darwin wrote some 6,200,000 words of science, of which roughly 45,000 (nearly $1 \%$ ) refer directly or indirectly to fishes. These have now been compiled, annotated, cross-referenced and elaborated on by Daniel Pauly in Darwin's Fishes, an encyclopedic treatment of these piscine works that the author proffers as "a belated entry" on Darwin's behalf.

As a youth, Darwin was an avid angler, stating in correspondence that: "On the rainy days I go fishing, on the good ones Entomologizing." During the voyage of the Beagle, he collected and preserved in alcohol dozens of fish species, about which he recorded notes in Fish in Spirits of Wine (the piscine part of his Catalogue, or master list, of trip collections). Immediately after the voyage, Darwin successfully encouraged the British naturalist and clergyman Leonard Jenyns — who had previously turned down Captain Fitzroy's original offer to be the Beagle's naturalist to complete the job of describing these fish specimens for science.

Later in life, Darwin incorporated his knowledge of fish into numerous works on topics that included the evolution of complex structures (such as electric organs in some rays and eels), sexual selection (in female-pregnant mollies and male-pregnant seahorses, for example) and the evolution of traits under artificial selection (as occurs in goldfish). Altogether, he refers to more than 250 fish species in his writings.

In Darwin's Fishes, Pauly describes everything that Darwin ever wrote on fish, the intent being to reveal how Darwin's world views were shaped by ichthyology, and vice versa. The book is encyclopedic not only in content but in style - its nearly 500 word entries (taxa or topics) are arranged alphabetically. A typical entry begins with a

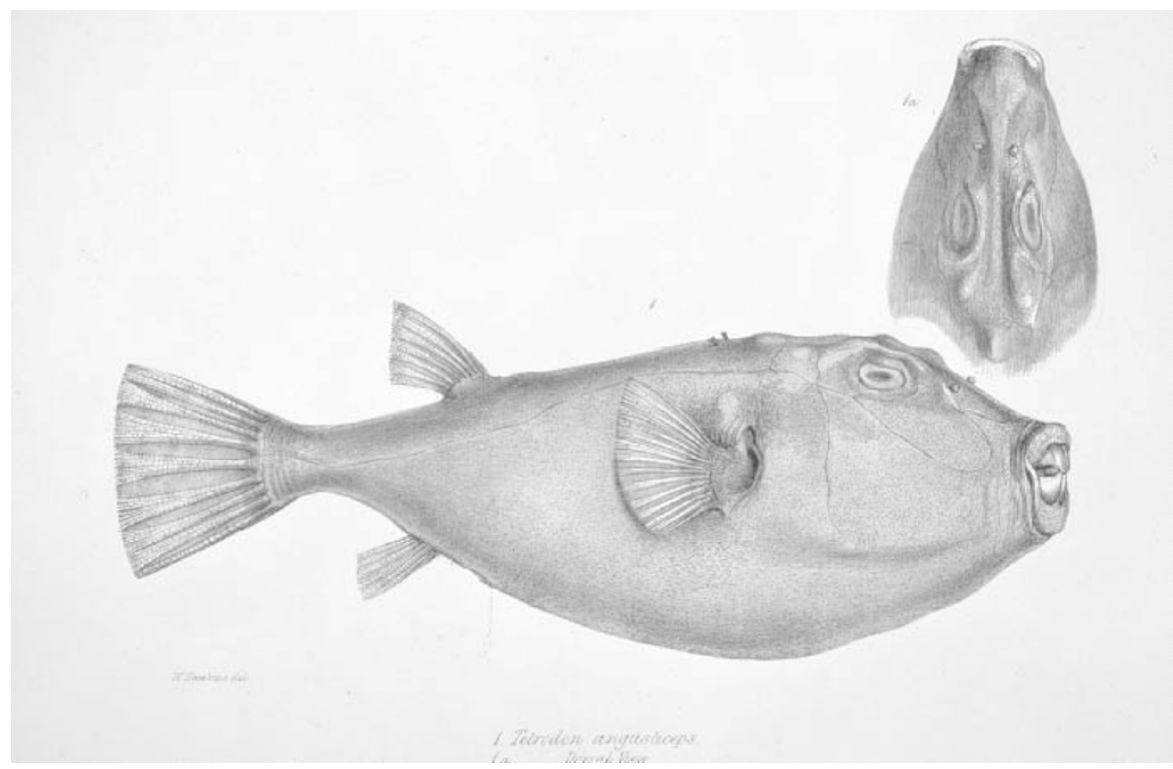

Fish list: Darwin catalogued species such as Sphoeroides angusticeps during the voyage of the Beagle.

definition, incorporates relevant quotations or references from Darwin's writings, describes how his thoughts on the subject were influenced by studies on fish, and places his ideas in the contexts of both his own era and modern times. Pauly frequently voices his own welcome opinions also. For example, in his commentary on how Darwin (and later evolutionists) grappled with explaining the evolution of complex eyes, Pauly states: "This is strange, as the eyes are the organs for which intermediate steps that increase the fitness of their owners are not only easy to conceive, but occur in a wide range of animals." And with regard to Darwin's words on the behaviour of nesting wrasses, Pauly concludes: "One cannot but wonder whether the reason why these male wrasses work together so nicely with the females on their domestic arrangements is because, as protogynous hermaphrodites, they can draw on insights from their previous life as females."

Darwin's Fishes is not the type of book to be read from cover to cover but is wonderful fun to dip into, even randomly. It is an eclectic, scholarly reference work, rich in historical content and chock full of interesting quotes and insights from Darwin and Pauly alike. Pauly has an engaging writing style and is not afraid to use humour and self-deprecation. In the preface, for example, he short-circuits potential criticisms when he writes: "Many sections of this book read like laundry lists. I have attempted to cover this up, mainly through levity, the result being that this book will probably irritate serious scholars, but still bore students to tears." These concerns have elements of truth, but are considerably overstated. This book is indeed an all-butthe-kitchen-sink 'chrestomathy' (you can find that word as an entry in Darwin's Fishes), but it is a delightful one.

John C. Avise is in the Department of Genetics,

University of Georgia, Athens, Georgia 30602, USA.

\section{Infinite beauty}

\section{Fractals and Chaos: The} Mandelbrot Set and Beyond by Benoit B. Mandelbrot Springer: 2004. 308 pp. \$49.95, £38.50, $€ 49.95$

\section{Kenneth Falconer}

It was just over 20 years ago that the Mandelbrot set took the world by storm. Pictures of extraordinary complexity and beauty appeared in scientific and glossy magazines, on the walls of art galleries and classrooms, on posters and even on tablemats. With the increasing availability of personal computers, drawing the Mandelbrot set became a standard exercise for those learning programming, and it was frequently an addiction for computer buffs, who were able to explore its intricacy by forever homing in on parts of the structure. Perhaps it is not surprising that such a simple procedure enabling almost anyone to produce an object of immense sophistication and attractiveness caught the public imagination.

The definition of the Mandelbrot set, denoted by $M$, is indeed extremely simple. Given a complex number $c$, start at the origin 0 and follow the trail of points obtained by repeatedly applying the transformation $f(z)=z^{2}+c$, that is, the sequence $0, c, c^{2}+c$, $\left(c^{2}+c\right)^{2}+c \ldots$ If these points never go far away from the origin then $c$ is in $M$, but if they wander off to infinity, $c$ is not in $M$. This straightforward check allows one to scan across a region of the complex plane to determine the extent of $M$.

Crude pictures of $M$ show a main cardioid surrounded by circular 'buds' of decreasing size. But more detailed investigation, pioneered by Benoit Mandelbrot in 1980, 


\section{The pen is mightier than the camera}

\section{Medical drawings bring a new dimension to the Royal Academy's Summer Exhibition in London.}

\section{Martin Kemp}

Why would anyone involved in the sciences resort to drawing by hand? In this era of digital photography, which generates high-resolution images that can be manipulated at will, and of devices such as electron microscopes that can be set up to see and represent an object without fallible human intervention, why use such an apparently outmoded technique?

The answer is that the act of accurate drawing is still the best way to guarantee intense looking on the part of the artist, and, in terms of communication with the viewer, it provides a visual language of unprecedented focus, directness and dynamism. A drawing can transcend temporal limitations in a way that no other static image can. Data from different stages in a process or procedure can be overlaid yet remain clear, and anyone witnessing the making of the drawing can be taken step-bystep through what is being demonstrated.

Any artist who draws, or anyone who has sat for a drawn portrait, will not need telling that drawing involves the most intense visual concentration. It is a way of "learning how to see", to quote the first president of the Royal Academy, Joshua Reynolds. It is appropriate that when David Hockney and Allen Jones, former enfants terribles of the 1960s, were asked to organize a section devoted to drawing at this year's Royal Academy Summer Exhibition, they decided to include examples from outside Fine Art.

The drawings that have created the greatest stir — and won a prize — are by the heart surgeon Francis Wells of Papworth Hospital in Cambridge, UK. The example shown here illustrates the repair of a post-myocardial infarction ventricular septal

reveals much, much more: the buds are all surrounded by smaller buds, which in turn support even smaller ones, and so on. Homing in on the boundary of $M$ reveals a menagerie of multi-branched spirals, dragons and seahorses. Hairs of imperceptible fineness extend from the buds, holding along their lengths minute replicas of the entire Mandelbrot set.

Is the Mandelbrot set just a pretty curiosity? Far from it. It is a fundamental parameter information about nonlinear processes. First, the position of a complex number $c$ relative to $M$ tells us a great deal about the iteration of the quadratic mapping $f(z)=z^{2}+c$. The (filled-in) 'Julia set' at $c$ consists of those complex numbers $z$ whose iterates under repeated application of $f(z)=z^{2}+c$ never wander far from the origin. This Julia set comprises a single piece precisely when $c$ lies in the Mandelbrot set. (Interestingly, this topological dichotomy was noted by Pierre set that encodes an enormous amount of

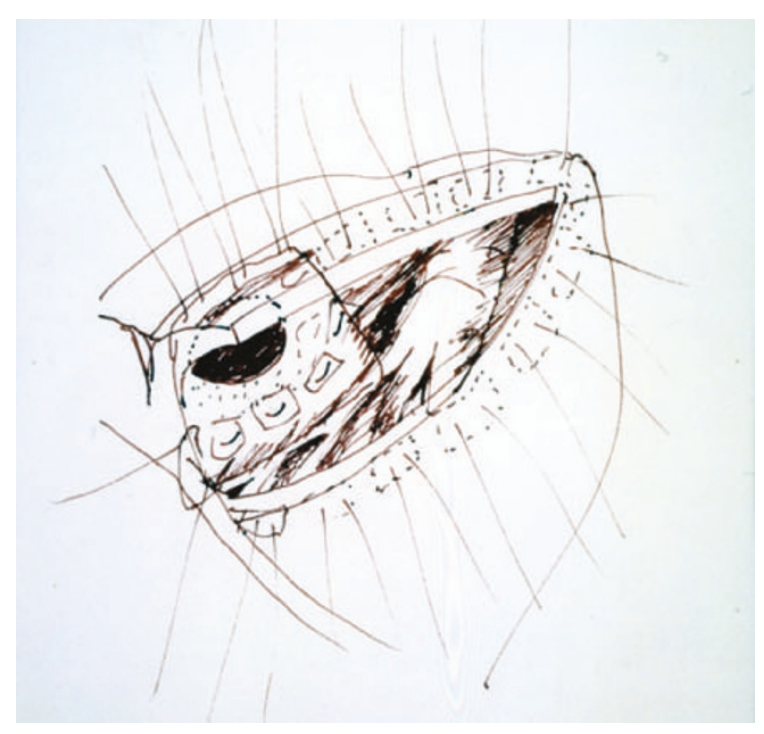

clutter that would be generated in a photograph or video. Even for someone who has not seen the drawing being made, the sense of the motion of the draughtsman's hand, in accordance with the flow of his ideas, is clear, just as it would be in a rapid sketch by Hockney or Jones. Indeed, Wells' sketch shares notable affinities with Hockney's drawings, especially those of the 1960s.

Wells himself is fully conscious of the special quality of the act of communicative drawing in time and place: "Having just completed the operation, drawing one's thoughts feels like an extension of the operation with the hand-eye interworking." This demonstrates defect. Wells explains that it illustrates "the incision in the left ventricle to get access to the ventricular cavities, the relationship of the papillary muscles and the underside of the atrioventricular valves, the lesions involved, in this case a hole in the septum, the placement of the sutures for the introduction of the patch to close the defect and the patch in place. Finally, the sutures necessary to close the incision are also shown."

The circumstances of its creation explain the impulsive character of the drawn line. This picture was drawn after the operation, over a cup of coffee in the surgeon's room, to explain the procedure to visiting and trainee surgeons. The speed and weight of line and the selective hatching are integral to the act of emphatic communication, picking out exactly what is essential, filtering out all the visual

Fatou and Gaston Julia in 1918-19, but it was many years before its real significance and delicacy was appreciated.)

Much more than this, the exact position of $c$ in $M$, such as the bud in which it lies, gives a very full description of how the iterates of $f(z)$ behave: for example, whether there are periodic cycles. Even more surprising is that, although defined in terms of the simplest of nonlinear maps, $f(z)=z^{2}+c$, the Mandelbrot set is 'universal' in that it underlies the behaviour of very large classes of more complicated nonlinear mappings, the likes of which crop up throughout modern mathematics and its applications.

The emergence of the Mandelbrot set in 1980 led to a flurry of activity among mathematicians trying to understand its structure and significance, resulting in some of the most impressive advances in pure mathematics in recent years. In 1982, Adrien Douady and John Hubbard proved the (far from trivial) fact that $M$ is connected, though "the superiority of drawing over other means of visual record, as it is a process of communicating thought and ideas as composed in the mind without the constraints of any equipment limitations."

It is interesting to witness moves to reintroduce drawing into the teaching of sciences and technologies. As Wells says, drawing is "powerful in teaching and can enhance explanation - acting out the move ... through the use of line. I feel this is very like the cognitive process in the artist, who will look and look before committing a line to paper."

The drawings are on show at the Summer Exhibition of the Royal Academy of Arts in London until 16 August.

Martin Kemp is professor of the history of art at the University of Oxford, Oxford OX1 1PT, UK, and co-director of Wallace Kemp Artakt. it is still unknown whether $M$ is locally connected - can you travel between nearby points of Mstaying inside $M$ without making too long a detour? In 1998, Mitsuhiro Shishikura showed that the boundary of $M$ has fractal dimension 2, which means that it is just about as complicated as can be, though it is still not known whether this boundary has positive area.

This is the fourth volume of Mandelbrot's Selecta, comprising edited reprints of the author's papers. Largely from the 1980s, these include the series of seminal papers that revealed the magnificence and omnipresence of the Mandelbrot set, together with other papers related to the iteration of functions. Several sections provide an overview of the work along with its scientific and historical background. One chapter has been written specifically to help the nonexpert appreciate the rest of the book.

Much of the material does not require particularly technical knowledge, so the 
book should be accessible to a wide readership. It provides a fascinating insight into the author's journey of seeing and discovering as the early pictures of the Mandelbrot set started to reveal a whole new world. It gives a feeling for his philosophy and approach of experimental mathematics - an approach that has changed the way we think about mathematics and science.

Kenneth Falconer is professor of pure mathematics at the School of Mathematics and Statistics, University of St Andrews, North Haugh, St Andrews, Fife KY16 9SS, UK.

Living machinery

\section{Bionanotechnology: Lessons \\ from Nature}

by David S. Goodsell

Wiley-Liss: 2004. 337 pp. £47.50, \$79.50,

$€ 66.70$

\section{Christof M. Niemeyer}

Nanotechnology is perfectly realized in biological systems. Cells are essentially biological assemblers that build thousands of custom-designed molecules and construct new assemblers. In Bionanotechnology, structural biologist David Goodsell describes what biology can teach us about engineering and manufacturing at the nanometre scale.

Goodsell kicks off with an introduction to the history of nanotechnology, which was pioneered by Richard Feynman and widely popularized by Eric Drexler's evocative idea of a self-replicating assembler building nanoscale devices atom by atom. He then takes a detailed look at the composition and structural principles of biomolecules harnessed in the cell, describing numerous bionanomachines in action, ranging from proteins, nucleic acids and membranes, to enzyme catalysis, the machinery of DNA transcription and translation, and biomolecular motors. The basic functional principles of nature's nanotechnology are elucidated, in particular the information-driven synthesis of biological molecules, the energetics and regulation of biological processes, and the traffic across membranes and signal transduction along them. The mechanistic aspects of biomaterials are also highlighted, for instance the interplay of myosin and actin filaments within the muscle sarcomere.

Throughout this fascinating journey, many fundamental principles are highlighted to introduce the reader to the unfamiliar world of nature's nanotechnology: the various bonding forces, thermal motion, the negligibility of gravity and inertia at the nanoscale, molecular recognition, selfassembly and biomolecular flexibility.

A survey of what is known about the general principles underpinning the structure and function of natural nanomachines is

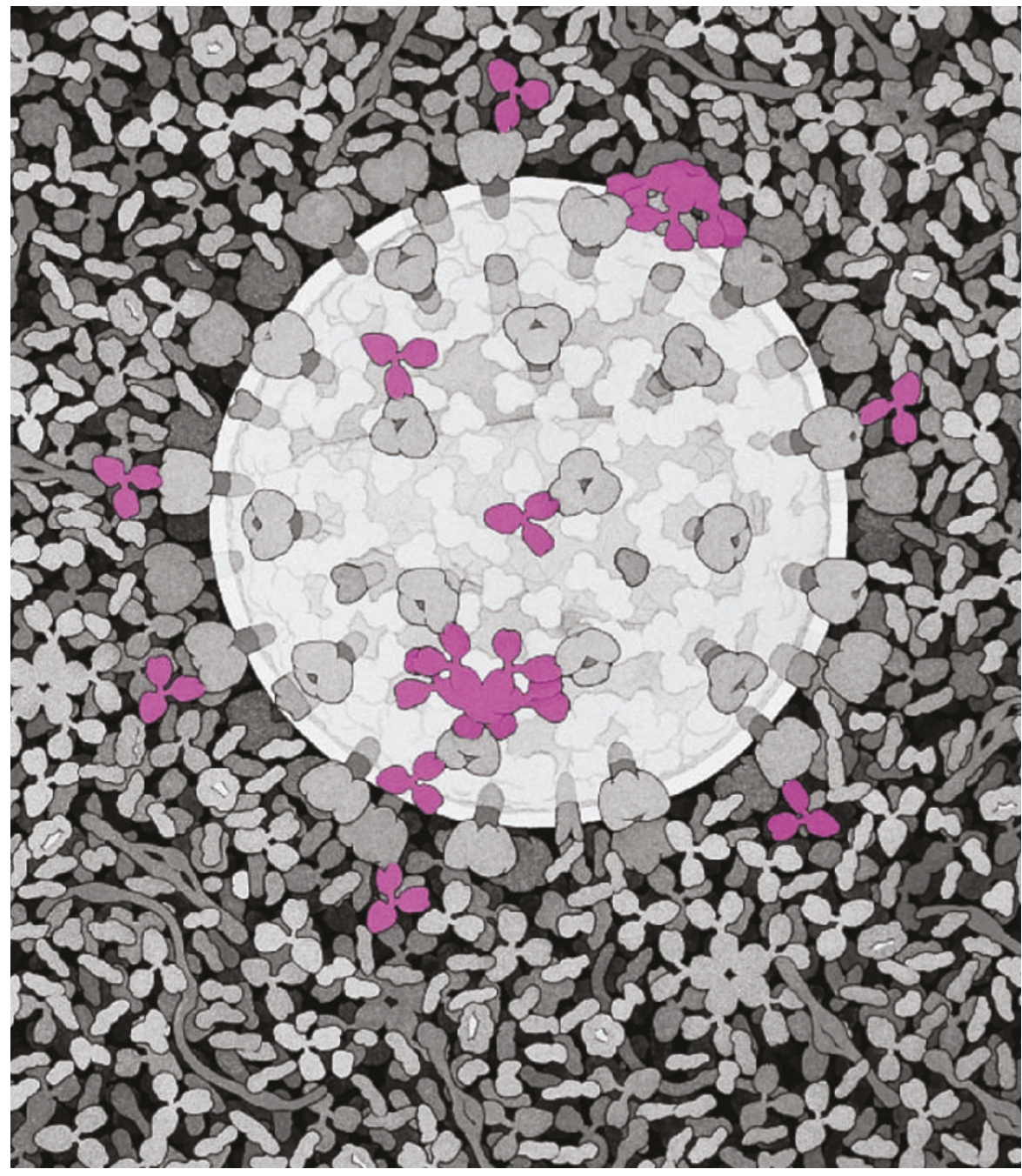

Small wonder: antibodies (purple) are just one example of the way nature uses nanotechnology.

rounded off by an overview of the techniques that biotechnology has at its disposal to harness and modify this machinery - for example, recombinant DNA and protein technology, biomolecular structure determination, modelling and the tailoring of proteins and nucleic acids by directed evolution. The author then describes exciting applications of bionanotechnology that were the subject of published research at the time of writing in mid-2002. These include projects involving nanomedicine, self-assembly at various length scales, harnessing biomolecular motors, DNA computers, artificial life and biomolecular/bio(in)organic hybrid materials.

In the final chapter, Goodsell speculates about the future of bionanotechnology. $\mathrm{He}$ uses the best currently available data to relate three futuristic case studies: a "nanotube synthase" (an enzyme that would build nanotubes); a nanoscale assembler (a ribosomelike device that places molecular fragments in three-dimensional space with full positional control at the nanometre scale); and autonomous nanorobots that would inspect all the cells in the human body for mutations in the cancer-associated $p 53$ gene.
Bionanotechnology, being a synonym for nanobiotechnology, is a rapidly growing field that encompasses contributions from various disciplines, ranging from engineering and computational sciences to physics, chemistry and biology. Hence, there is an increasing need for a didactic textbook that provides a thorough introduction to biomolecular sciences and their impact on nanotechnology. Godsell's book is the first to meet this demand.

Written in the style of an excellent biochemistry textbook, Bionanotechnology points the reader to general principles of the biological nanoworld, and thus provides readers with guidance on the design of their own devices and systems. The intention of this book is to invite further reading, so one should not expect too many technical details taken from the growing literature in this field. There are many graphic illustrations of bionanomachines, although it is a pity that these were not reproduced in full colour. I can highly recommend this book. I enjoyed reading every single page. Christof M. Niemeyer is professor of chemistry at the University of Dortmund, Otto-Hahn Strasse 6, 44227 Dortmund, Germany. 